\title{
Friction-stir welding of dissimilar AA 2024-T3 to AZ31B-H24 alloys
}

\author{
X. Cao • M. Jahazi
}

Received: 2 July 2009 /Accepted: 19 October 2009/Published online: 31 October 2009

(C) Springer-Verlag London Limited 2009

This article has been retracted because of copyright issues that cannot be resolved.

This article has been retracted because of copyright issues that cannot be resolved.

X. Cao $(\bowtie) \cdot$ M. Jahazi

Aerospace Manufacturing Technology Centre,

Institute for Aerospace Research,

National Research Council Canada,

5145 Decelles Ave,

Montreal, QC H3T 2B2, Canada

e-mail: xinjin.cao@cnrc-nrc.gc.ca 